\title{
ZOOPLÂNCTON DE UM LAGO URBANO DOMINADO PELA MACRÓFITA PISTIA STRATIOTES L. (ARACEAE)
}

\section{Lidiane Cristina da Silva ${ }^{1}$}

Natalia Felix Negreiros ${ }^{2}$

\section{Renata Martins dos Santos ${ }^{3}$}

Resumo: Os lagos urbanos estão submetidos a grandes impactos causados pelas atividades antrópicas desenvolvidas no seu entorno, o que geralmente causa impacto sobre o corpo d'água, entre estes estão as florações de cianobactérias e de macrófitas aquáticas. A distribuição e estrutura do zooplâncton em ecossistemas de água doce são influenciadas pelo tipo de habitat, em especial quando criado por várias espécies de macrófitas. Este rápido inventário tem como objetivo analisar qualitativa e quantitativamente a comunidade zooplanctônica que caracteriza o Lago do Parque Ecológico do Baguaçu, a fim de complementar a avaliação da qualidade da água e detectar os efeitos das macrófitas sobre esta comunidade. Foram registradas as medidas da temperatura da água, $\mathrm{pH}$, condutividade, transparência da água. A concentração de clorofila a foi determinada pelo método de extração com acetona $90 \%$ a frio. $\mathrm{O}$ zooplâncton foi amostrado com rede de plâncton. As amostras foram fixadas com formol na concentração final $4 \%$ e os organismos foram identificados e contados, calculando-se então a densidade e os índices de diversidade, dominância e equitabilidade. Foram identificadas 31 espécies, sendo que para Protozoa foi

\footnotetext{
${ }^{1}$ Bióloga, Programa de Pós-Gradução em Ecologia e Recursos Naturais, Universidade Federal de São Carlos. lidianecris@yahoo.com.br

${ }^{2}$ Bióloga, Programa de Pós-Gradução em Ecologia e Recursos Naturais, Universidade Federal de São Carlos. natalia felix@yahoo.com.br

${ }^{3}$ Bióloga, Programa de Pós-Gradução em Ecologia e Recursos Naturais, Universidade Federal de São Carlos. renata cordis@yahoo.com.br
} 
registrado a maior riqueza (13 espécies), Rotifera e Meroplâncton (7 espécies cada) e Copepoda e Cladocera (2 espécies cada). A análise da comunidade zooplanctônica do Lago do PEBA revelou um padrão diferenciado na abundância e diversidade de espécies para os distintos grupos taxonômicos. Os protozoários e rotíferos dominaram tanto em termos de riqueza quanto em densidade, sendo estes favorecidos pelo micro-hábitat proporcionado pelas macrófitas.

Palavras-chave: lago urbano, eutrofização, diversidade de espécies.

\section{Introdução}

Os lagos urbanos estão submetidos a grandes impactos causados pelas atividades antrópicas desenvolvidas no seu entorno, e geralmente a qualidade de suas águas está intimamente ligada às condições econômicas e de saneamento dos grandes centros. A gestão destes ambientes constitui um grande desafio para administradores e tomadores de decisão em diferentes esferas do poder municipal ou estadual. Nas últimas décadas os estudos das comunidades aquáticas têm sido incluídos nos protocolos de avaliação de impactos ambientais e dentre estas a comunidade zooplanctônica tem sido frequentemente utilizada.

A comunidade zooplanctônica pode ser considerada como um compartimento estratégico na dissipação energética dos ecossistemas aquáticos e na manutenção e orientação das teias tróficas aquáticas. O seu posicionamento na cadeia alimentar, com um alto grau de conexão com os produtores primários, a torna extremamente suscetível às mudanças estruturais ocorridas neste nível trófico inferior. Em função do rápido ciclo de vida, as alterações ocorridas na comunidade fitoplanctônica são rapidamente espelhadas pelo zooplâncton, que passa a sinalizar a intensidade e as condições estabelecidas durante e após a consolidação destas perturbações. Além disso, o zooplâncton também apresenta forte vinculação com os níveis tróficos mais elevados da teia trófica aquática (SORANNO et al., 1985), sendo responsável por uma importante rota de transferência de energia dos produtores primários aos peixes. Todas essas características tornam a comunidade zooplanctônica elemento-chave para o entendimento das modificações ocorridas em ecossistemas aquáticos particularmente devido à eutrofização. 


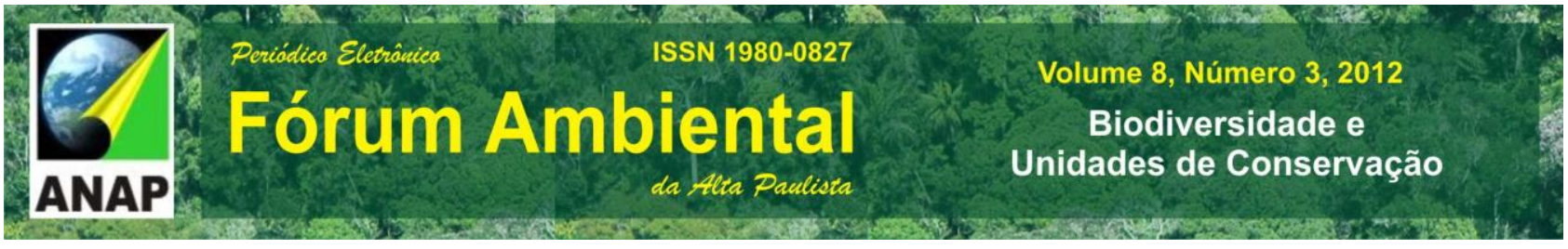

Esta comunidade é formada por organismos que apresentam diferentes estratégias de desenvolvimento, relacionadas à reprodução e alimentação, possibilitando a colonização de ambientes com distintas características físicas e químicas. Esta é constituída principalmente por protozoários, rotíferos, cladóceros e copépodos (WETZEL, 1983). Este conjunto de organismos pode apresentar grande variedade de hábitos alimentares. Os organismos zooplanctônicos apresentam tamanhos de poucos micrômetros até alguns milímetros, fato este, que contribui para a diversidade estrutural do ambiente (BOZELLI; HUSZAR, 2003).

A distribuição do zooplâncton em ecossistemas de água doce é conhecida tanto na zona limnética quanto na litorânea. Além disso, o tipo de habitat ecológico, em especial quando criado por várias espécies de macrófitas, tem influência sobre a estrutura da comunidade zooplanctônica (KUCZYŃSKA-KIPPEN, 2007). No entanto, a presença de bancos de macrófitas além de influenciar a estrutura da comunidade zooplanctônica, pode ter um impacto sobre os diversos fatores ambientais (ROSENZWEIG, 1991; MOORE et al., 1994). As macrófitas oferecem um refúgio para o zooplâncton contra peixes planctívoros (LAURIDSEN et al., 1996), bem como contra a predação por invertebrados.

Segundo Wetzel (1983), a grande maioria dos lagos no mundo são pequenos e rasos, com características morfométricas que propiciam o desenvolvimento de extensas comunidades de macrófitas aquáticas em suas regiões litorâneas, as quais desempenham importante papel no metabolismo de todo o sistema. Assim, macrófitas e sua microflora e microfauna associadas, podem exercer influência importante na dinâmica trófica dos ecossistemas lacustres.

Este rápido inventário vem analisar qualitativa e quantitativamente a comunidade zooplanctônica que caracteriza o Lago do Parque Ecológico do Baguaçu, a fim de complementar a avaliação da qualidade da água e detectar os efeitos das macrófitas sobre esta comunidade.

\section{Área de estudo}

O Parque Ecológico do Baguaçu (PEBA) (2113'07”.5 S e 50²5’37”.5 W) está localizado na cidade de Araçatuba, SP, (ARAÇATUBA, 1978) e corresponde a uma 
reserva ecológica de visitação pública. O lago existente neste parque tem como principal efluente o Ribeirão Baguaçu que deságua no rio Tietê. O Ribeirão Baguaçu abastece $70 \%$ da cidade de Araçatuba e o volume de água captado é de aproximadamente $35 \%$ de sua capacidade. Este lago apresenta uma profundidade média estimada na faixa de 3,5 a 5,0 metros, com presença de macrófitas aquáticas, com dominância de Pistia stratiotes, popularmente conhecida como alface d'água, e também florações de microalgas.

\section{Materiais e Métodos}

\section{Variáveis abióticas}

A coleta foi realizada no dia 23 de setembro de 2012 em um ponto localizado na região litorânea do Lago do Parque Ecológico do Baguaçu, com profundidade de $70 \mathrm{~cm}$.

A temperatura da água foi mensurada com termômetro de mercúrio, a condutividade elétrica, $\mathrm{pH}$ e concentração de oxigênio dissolvido foram aferidos por um condutivímetro digital, um pHmetro portátil e um oxímetro respectivamente. A transparência da água foi determinada através da realização de leituras do desaparecimento e reaparecimento visual do disco de Secchi. Para calcular o limite da zona eufótica o valor da transparência da água foi multiplicado pelo coeficiente empírico igual a 2,7 (MARGALEF, 1983). A concentração do material em suspensão foi determinada segundo o método gravimétrico descrito em Teixeira et al. (1965).

\section{Variáveis bióticas}

Os organismos foram coletados com rede de plâncton com diâmetro de $30 \mathrm{~cm}$ e abertura de malha de $68 \mu \mathrm{m}$, filtrando-se 15 litros de água. Posteriormente foram fixados com formol 4\%. A identificação e quantificação dos microcrustáceos (Cladocera e Copepoda) foram feitas em placas de acrílico quadriculadas sob microscópio estereoscópio (aumento até 50x) contando sub-amostras ou, até a amostra inteira, para os organismos raros. Os rotíferos foram contados em câmara de Sedgwick-Rafter sob 
microscópio óptico (aumento até 1000x, com ocular graduada). Para a identificação utilizou-se bibliografia especializada. Com os dados, pode-se calcular a densidade e a abundância das espécies. Os índices de diversidade, dominância e equitabilidade foram calculados utilizando-se o programa estatístico PAST version 1.94 (HAMMER et al., 2001).

\section{Resultados e discussão}

Os valores das características físicas e químicas da água no Lago do Parque Ecológico do Baguaçu no ponto de coleta estão apresentados na Tabela 1.

Tabela 1: Características físicas e químicas e biológicas da água do Lago do Parque Ecológico do Baguaçu no ponto de coleta.

\begin{tabular}{cc}
\hline & PEBA \\
\hline Profundidade $(\mathbf{c m})$ & 70 \\
pH & 23 \\
Temperatura da água $\left({ }^{\circ} \mathbf{C}\right)$ & 6,3 \\
Oxigênio dissolvido $\left(\mathbf{m g} \cdot \mathrm{L}^{-1}\right)$ & 5,6 \\
Condutividade elétrica $\left(\boldsymbol{\mu S} \cdot \mathbf{c m}^{-1}\right)$ & 23 \\
Secchi $(\mathbf{c m})$ & 50 \\
Material em suspensão total $\left(\mathbf{m g} \cdot \mathrm{L}^{-1}\right)$ & 0,0073 \\
Material orgânico $\left(\mathbf{m g} \cdot \mathrm{L}^{-1}\right)$ & 0,0052 \\
Material inorgânico $\left(\mathbf{m g} \cdot \mathrm{L}^{-1}\right)$ & 0,0021 \\
\hline
\end{tabular}

A temperatura da água foi relativamente alta, como esperado para o período de coleta caracterizado como um período seco. $\mathrm{O}$ pH manteve-se ácido com valor menor que 6,0 . A concentração de oxigênio dissolvido registrada está dentro da faixa registrada para outros lagos urbanos no Brasil (MELÃO, 1997). Os valores da condutividade elétrica foram baixos. A transparência da água foi total devido a pequena profundidade do local de coleta. A quantidade de material em suspensão foi baixa e houve predominância de matéria orgânica, o que indica um ambiente com alta taxa de decomposição.

Os parâmetros físicos e químicos analisados mostraram que o Lago do PEBA é um corpo de água pouco profundo, com águas ácidas devido à presença de grande 
quantidade de ácidos húmicos, que dá uma coloração escura à água. Apresenta uma boa transparência, o que pode favorecer os produtores neste ambiente, possibilitando desta forma alimento de qualidade e em quantidade para o zooplâncton.

Foram identificadas 31 espécies para a comunidade zooplanctônica (Tabela 2). No grupo dos protozoários foi registrado o maior número de espécies (13), além de uma subespécie. Rotifera e os organismos meroplanctônicos foram representados por sete táxons. A menor riqueza foi registrada para os microcrustáceos Cladocera e Copepoda, ambos sendo representados por apenas duas espécies.

Tabela 2: Lista das espécies zooplanctônicas registradas no Lago do Parque Ecológico do Baguaçu.

\begin{tabular}{|c|}
\hline Cladocera \\
\hline Leydigiopsis megalops \\
\hline Simocephalus latirostris \\
\hline Copepoda \\
\hline Cyclopoida \\
\hline Copepoditos \\
\hline Náuplios \\
\hline Harpacticoida \\
\hline Náuplios \\
\hline Rotifera \\
\hline Brachionus falcatus \\
\hline Dipleuchlanis propatula \\
\hline Lecane curvicornis \\
\hline Lecane leontina \\
\hline Platyias quadricornis \\
\hline Polyarthra aff. vulgaris \\
\hline Rotaria neptunia \\
\hline Protozoa \\
\hline Arcella hemisphaerica \\
\hline Arcella hemisphaerica undulata \\
\hline Arcella megastoma \\
\hline Arcella mitrata \\
\hline Arcella vulgaris \\
\hline Astramoeba sp. \\
\hline Centropyxis aculeata \\
\hline Ciliado \\
\hline Cyphoderia ampulla \\
\hline
\end{tabular}




\begin{tabular}{c} 
Difflugia corona \\
Difflugia cylindrus \\
Difflugia limnetica \\
Difflugia lobostoma \\
Paradileptus elephantinus \\
\hline Meroplâncton \\
\hline Bryozoa \\
Chironomidae \\
Hydracarina \\
Gastrotricha \\
Nematoda \\
Oligochaeta \\
Ostracoda
\end{tabular}

Os representantes dos cladóceros são pertencentes a duas famílias distintas, Leydigiopsis megalops da família Chydoridae e Simocephalus acutirostris da família Daphnidae. As espécies da família Chydoridae são típicos de região litorânea e encontrados muitas vezes associados aos bancos de macrófitas (SANTOS-WISNIEWSKI et al., 2002).

Para o grupo Copepoda foram registradas apenas as formas juvenis (náuplios e copepoditos) da Ordem Cyclopoida e Harpacticoida. Alguns trabalhos em água doce revelam a importância das formas jovens de Copepoda na estrutura da comunidade zooplanctônica devido a grande densidade em que ocorrem (ROBERTSON; HARDY, 1984; NUNES et al., 1996). Sabe-se que o ciclo de vida dos Copepoda é longo, comparativamente ao dos demais componentes do zooplâncton (Rotifera e Cladocera), o que os tornam suscetíveis a uma alta taxa de mortalidade antes de atingirem o estágio adulto. Somado a isto, quando adultos recebem grande pressão de predação que, em conjunto, pode justificar a predominância das formas juvenis de Copepoda na comunidade zooplanctônica. A Ordem Harpacticoida é raramente encontrada no plâncton, sendo sua ocorrência relacionada ao sedimento (ANDRADE, 2007).

Rotifera foi o segundo grupo em termos de riqueza se espécies e esta maior riqueza para este grupo é um padrão recorrente em ambientes aquáticos tropicais (MATSUMURA-TUNDISI, 1999). Rocha et al. (1995) apresentam uma relação da riqueza de 22 diferentes ecossistemas de água doce e afirmam que a riqueza de rotíferos é 
amplamente dependente das famílias Lecanidae e Brachionidae, semelhante ao observado no presente estudo. As famílias encontradas no Lago do PEBA apresentam elevada riqueza espécies em regiões tropicais. Green (1972) afirma que o gênero Lecane é reconhecidamente típico das regiões litorâneas de lagoas.

Os protistas possuem papel ecológico muito importante em sistemas naturais, pois constituem um dos principais componentes dos ciclos biogeoquímicos globais, regulando o suprimento e a demanda de carbono orgânico, a ciclagem de nutrientes e o balanço de gás carbônico e oxigênio dentro e entre os ecossistemas (SILVA, 2008). No presente estudo, estes organismos deste grupo foram os mais diversos e abundantes, no entanto, Auer e Arndt (2001) relatam que existe pouca informação sobre a influência do estado trófico do ambiente na estrutura taxonômica e na distribuição dos protistas em sistemas naturais.

Protozoa são raramente incluídos em estudo das comunidades zooplanctônicas de água doce. Isso ocorre, pois os cladóceros, copépodos e rotíferos são considerados os mais importantes em termos de densidade, biomassa, produção, grazing e ciclagem de nutrientes (HUTCHINSON, 1967). Além disso, os protozoários requerem um método de diferente daquele realizado na amostragem em estudos do zooplâncton (PACE; ORCCUT, 1981).

O padrão dos valores de densidade seguiu a tendência da riqueza, com Protozoa e Rotifera representando os maiores valores (Tabela 3 e Figura 1). Estes grupos são considerados $r$ estrategistas e possuem menores tamanhos em comparação aos demais representantes da comunidade zooplanctônica. Geralmente, ambientes com curtos tempo de residência favorecem o desenvolvimento desses indivíduos, como observado no reservatório do Broa (MATSUMURA-TUNDISI; TUNDISI, 1986); na represa de Barra Bonita (MATSUMURA-TUNDISI et al., 1990; ESPÍNDOLA, 1994; SANTOS-WISNIEWSKI, 1998) e no reservatório do Monjolinho (NOGUEIRA, 1990; OKANO, 1994). Outro fato relevante para esses dois grupos está relacionado à eutrofização. Alguns padrões têm sido observados em corpos d' água brasileiros onde Rotifera e Protozoa são registrados em ambientes eutróficos enquanto Copepoda e Cladocera são mais abundantes que os demais em ambientes oligotróficos (MATSUMURA-TUNDISI et al., 1999). 
O microzooplâncton (rotíferos e protozoários) dominou sobre os microcrustáceos. Esse padrão de distribuição particular dos grupos zooplanctônicos é típico de ecossistemas de água doce e refletem as relações entre os organismos planctônicos na pirâmide trófica.

Tabela 3: Densidade das espécies zooplanctônicas registradas no Lago do Parque Ecológico do Baguaçu.

\begin{tabular}{|c|c|}
\hline Cladocera & PEBA \\
\hline Leydigiopsis megalops & 2400 \\
\hline Simocephalus latirostris & 218 \\
\hline Total Cladocera & 2618 \\
\hline \multicolumn{2}{|l|}{ Copepoda } \\
\hline \multicolumn{2}{|l|}{ Cyclopoida } \\
\hline Copepoditos & 84000 \\
\hline Náuplios & 72000 \\
\hline \multicolumn{2}{|l|}{ Harpacticoida } \\
\hline Copepoditos & 2400 \\
\hline Náuplios & 12000 \\
\hline Total Copepoda & 170400 \\
\hline \multicolumn{2}{|l|}{ Rotifera } \\
\hline Brachionus falcatus & 24000 \\
\hline Dipleuchlanis propatula & 12000 \\
\hline Lecane curvicornis & 24000 \\
\hline Lecane leontina & 12000 \\
\hline Platyias quadricornis & 12000 \\
\hline Polyarthra aff. vulgaris & 12000 \\
\hline Rotaria neptunia & 60000 \\
\hline Total Rotifera & 156000 \\
\hline \multicolumn{2}{|l|}{ Protozoa } \\
\hline Arcella hemisphaerica & 60000 \\
\hline Arcella hemisphaerica undulata & 12000 \\
\hline Arcella megastoma & 60000 \\
\hline Arcella mitrata & 24000 \\
\hline Arcella vulgaris & 108000 \\
\hline Astramoeba sp. & 36000 \\
\hline Centropyxis aculeata & 120000 \\
\hline Ciliado & 132000 \\
\hline Cyphoderia ampulla & 48000 \\
\hline Difflugia corona & 24000 \\
\hline
\end{tabular}




\begin{tabular}{c|c} 
Difflugia cylindrus & 12000 \\
Difflugia limnetica & 36000 \\
Difflugia lobostoma & 60000 \\
Paradileptus elephantinus & 12000 \\
Total Protozoa & 744000 \\
\hline Meroplâncton & \\
\hline Bryozoa & 24000 \\
Chironomidae & 343 \\
Hydracarina & 218 \\
Gastrotricha & 12000 \\
Nematoda & 84000 \\
Oligochaeta & 1029 \\
Ostracoda & 800 \\
Total Meroplâncton & 122390 \\
\hline Total geral & $\mathbf{1 1 9 5 4 0 8}$ \\
\hline
\end{tabular}

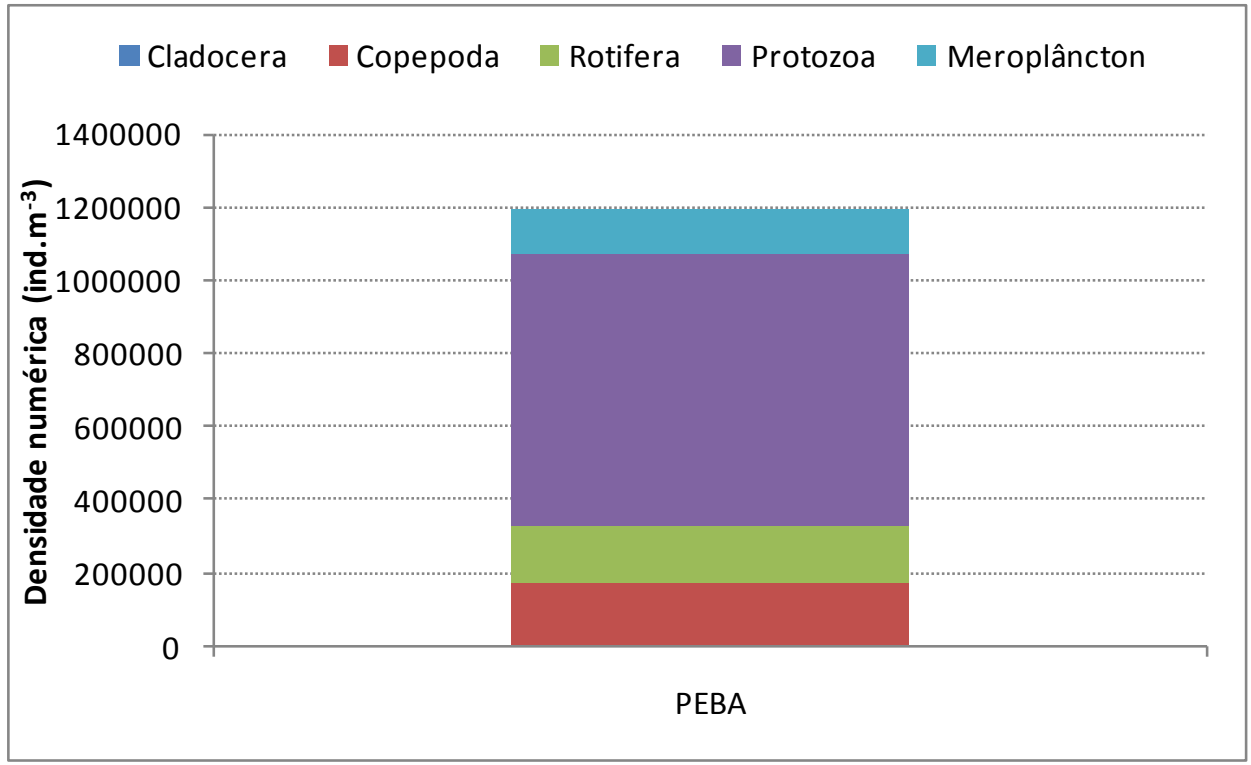

Figura 1. Densidade (ind. $\mathrm{m}^{-3}$ ) dos grupos zooplanctônicos no Lago do Parque Ecológico do Baguaçu.

A grande abundância de protozoários e rotíferos nas macrófitas pode estar relacionada principalmente com a presença de perifiton, que durante a seca encontram neste local um ótimo substrato para se desenvolver. Foi observada a presença de Simocephalus latirostris associada também com as macrófitas, provavelmente este 
cladócero de maior tamanho poderia estar utilizando as macrófitas como refúgio contra peixes predadores (LAURIDSEN; LODGE, 1996).

O índice de diversidade e de equitabilidade da comunidade zooplanctônica no Lago do PEBA foram altos (3,02 e 0,85 respectivamente), consequentemente o índice de dominância foi baixo (0,06). Segundo Rosso (1996) o índice de Shannon-Wiener, já tradicionalmente designado como índice de Shannon, é a medida de diversidade mais consagrada.

\section{Conclusões}

A análise da comunidade zooplanctônica do Lago do PEBA revelou um padrão diferenciado na abundância e diversidade de espécies para os determinados grupos taxonômicos. Os protozoários e rotíferos dominaram tanto em termos de riqueza quanto em densidade, sendo estes favorecidos pelo micro-hábitat proporcionado pelas macrófitas.

\section{Referências}

ANDRADE, L. P. Distribuição espacial e temporal da comunidade de crustáceos de águas intersticiais de um igarapé amazônico e um riacho da Mata Atlântica. 2007. 71 f. Tese (Doutorado em Ciências) - Instituto de Biociências, Universidade de São Paulo, São Paulo. 2007.

ARAÇATUBA. Mapa altimétrico: ano 1978. Araçatuba: Secretaria de Planejamento, Prefeitura Municipal de Araçatuba, 1978.

AUER, B.; ARNDT, H. Taxonomic composition and biomass of heterotrophic flagellates in relation to lake trophy and season. Freshwater Biology, v. 46, n.7, p. 959-972, 2001.

BOZELLI, R. L.; HUSZAR, V. L. M. Comunidades Fito e zooplanctonicas continentais em tempo de avaliação. Limnotemas. Sociedade Brasileira de Limnologia, 2003.

ESPINDOLA, E. L. G.1994. Dynamic of congeneric species of Notodiaptomus (Copepoda Calanoida) from Barra Bonita Reservoir, São Paulo. 1994. 336 p. Tese (Doutorado em Ciências da Engenharia Ambiental) Escola de Engenharia de São Carlos, Universidade de São Paulo, São Carlos, 1994.

GREEN, J. Freshwater ecology in the Mato Grosso, Central Brazil. III. Associations of Rotifera in meander lakes of the Rio Suiá Missú. Journal of Natural History, v. 6, p. 229-241, 1972.

HAMMER, U. T.; HARPER, D.; RYAN, P.2001. PAST: Paleontological statistics software package for education and data analysis. Paleontological Electronic, v. 4, n. 1, 9 p. 
HUTCHINSON, G. E. 1967. A Treatise on Limnology. II. Introduction to lake biology and their limnoplankton. New York: John Wiley \& Sons, 1967. 1115 p.

KUCZYŃSKA-KIPPEN N. M.; NAGENGAST B. The influence of the spatial structure of hydromacrophytes and differentiating habitat on the structure of rotifer and cladoceran communities. Hydrobiologia, v. 559, p. 203-212, 2006.

LAURIDSEN, T. L.; LODGE, D. M. Avoidance by Daphnia magna of fish and macrophytes: chemical cues and predator-mediated use of macrophyte habitat. Limnology Oceanography, v. 41, p. 794-798, 1996.

LAURIDSEN, T. L.; PEDERSEN, L. J.; JEPPESEN, E.; SONDERGAARD, M. The importance of macrophyte bed size for cladoceran composition and horizontal migration in a shallow lake. Journal of Plankton Research, v. 18, p. 2283-2294, 1996.

MARGALEF, R. Limnologia. Barcelona: Omega, 1983. 1100 p

MATSUMURA-TUNDISI, T. Diversidade de zooplâncton em represas do Brasil. In: HENRY, R. (Ed.). Ecologia de reservatórios: estrutura, função e aspectos sociais. Botucatu: FUNDBIO/FAPESP, 19990. p. 3954.

MATSUMURA-TUNDISI, T.; TUNDISI, J. Preliminary studies in a lacustrine environment. I. Preliminary data on zooplankton ecology of Broa Reservoir. Oecology, v. 25, p. 265-270, 1986.

MATSUMURA-TUNDISI, T.; LEITÃO, S. N.; AGUENA, L. S.; MIYAHARA, J. Eutrofização da represa de Barra Bonita: estrutura e organização da comunidade de Rotífera. Revista Brasileira de Biologia, v. 50, n. 4, p. 923-935, 1990.

MELÃO, M. G. G., 1997. A comunidade planctônica fitoplâncton e zooplâncton) e produtividade secundária do zooplâncton de um reservatório oligotrófico. 1997. 152 p. Tese (Doutorado em Ciências) - Universidade Federal de São Carlos, São Carlos,1997.

MOORE, B. C.; Funk, W. H.; Anderson, E. Water quality, fishery and biologic characteristic in a shallow, eutrophic lake with dense macrophyte populations. Lake Reservoir Management, v. 8, n. 2, p. 175-188, 1994.

NOGUEIRA, M. G. 1990. Dinâmica das populações planctônicas e fatores físico-químicos de um pequeno sistema artificial raso (represa do Monjolinho, São Carlos, SP). 1990. 244 p. Tese (Mestrado em Ciências da engenharia ambiental) - Escola de Engenharia de São Carlos, Universidade de São Paulo. 1990.

NUNES, M. A.; LANSAC - TÔHA, F. A.; BONECKER, C. C.; ROBERTO, M. C.; RODRIGUES, L. Composição e abundância do zooplâncton de duas lagoas do Horto Florestal Dr. Luiz Teixeira Mendes, Maringá, Paraná. Acta Limnologica Brasiliensia, v. 8, p. 207-219, 1996.

OKANO, W.Y. 1994. Análise da estrutura e dinâmica populacional da comunidade zooplanctônica de um reservatório artificial (Represa do Monjolinho, São Carlos - SP). Tese de Doutorado. Universidade Federal de São Carlos, São Carlos, SP, Brasil. 128p.

PACE, M. L.;. ORCUTT, J. R. Relative importanceof protozoans, rotifers and crustaceans in afreshwater zooplankton community. Lirnnology Oceanography, v. 26, p. 822-830, 1981.

ROBERTSON, B. A.; HARDY, E. R. Zooplankton of Amazonian lakes and rivers. The Amazon. Monographic Biological, v. 56, p. 337-352, 1984. 
ROCHA, O.; SENDACZ, S.; MATSUMURA-TUNDISI, T. Composition, biomass and productivity of zooplankton in natural lakes and reservoirs of Brazil. In: TUNDISI, J. B.; BICUDO, C. E.; MATSUMURATUDISI, T. (Eds.). Limnology in Brazil. Rio de Janeiro: ABC/SLB. p.151-165. 1995.

ROSENZWEIG, M. L. Habitat selection and population interactions: the search for mechanism. The American Naturalist, v. 137, p. 5-28, 1991.

ROSSO, S. Amostragem, repartição espacial e diversidade/dominância de comunidades de costões rochosos: uma abordagem metodológica. Laboratório de Ecologia Marinha/USP. 1996. 30 p.

SANTOS-WISNIEWSKI, M. J. Distribuição espacial e produção secundária da comunidade zooplanctônica do Reservatório de Barra Bonita - SP. 1998. 240 p. Tese (Doutorado em Ciências) - Universidade Federal de São Carlos, São Carlos.1998.

SANTOS-WISNIEWSKI, M. J.; ROCHA, O.; GÜNTZEL, A. M.; MATSUMURA-TUNDISI, T. Cladocera Chydoridae of high altitude water bodies (Serra da Mantiqueira), in Brazil. Revista Brasileira de Biologia = Brazilian Journal of Biology, v. 62, n. 4a, p. 681-687, 2002.

SILVA, M. B. Assembléias de amebas testáceas (Amoebozoa: Rhizopoda) associadas a rizosfera de Eichhornia crassipes (Martius) Solomons (Pontederiaceae) no Rio Cachoeira, Bahia. 2008. 115 f. Tese (Mestrado em Sistemas Aquáticos Tropicais) - Universidade Estadual de Santa Cruz, Ilhéus, BA. 2008.

SORANNO, P. A.; CARPENTER, S. R.; HE, X. 1985. Zooplankton biomass and body size. Pp 172-188. In: Carpenter, S. R.; Jitchell, F. (Eds.). The Trophic Cascade in Lakes. London: Cambridge Pergamon Press, 1985, $381 \mathrm{p}$.

TEIXEIRA, C.; TUNDISI, J. G; KUTNER, M. B. Plankton studies in a mangrove II. The standing stock and some ecological factors. Boletim do Instituto Oceanografia, v.24, p. 23-41, 1965.

WETZEL, R. G. Limnologia. 2. ed. Lisboa: Fundação Calouste Gulbenkian, 1983, 1110 p. 